\title{
Experiencia en intubación submandibular en pacientes con fracturas panfaciales
}

\section{Experience in submandibular intubation in patients with panfacial fractures}

Nelson Daniel Sánchez A. MD¹, Jimmy Bolaños J. OD. Esp. Mg², Luis Alberto Palacios C. MD. Esp³

\begin{abstract}
Objectives: To describe our experience in the implementation of the submandibular intubation technique in patients with panfacial fractures. Materials and Methods: Descriptive observational study, series of cases of patients with surgical reduction of panfacial fractures in whom the airway was secured by submandibular intubation, from July 2017 to October 2019. Results: 30 submandibular intubation procedures were recorded in 29 men and 1 woman for panfacial fractures. Fractures of the naso orbital ethmoidal complex were the most involved $(83.3 \%, n=25)$. In the execution, the average time was 7.7 minutes ( 5 to 10 minutes), there were only 2 cases of rupture of the pilot of the ball and desaturation, with rapid replacement without consequences. There were no early or late complications related to the surgical wound. Conclusions: The submandibular approach to secure the airway is an alternative with less technical difficulty and few complications. For his choice, the patient must be individualized. The surgical team must have clear communication and knowledge of the technique.
\end{abstract}

\section{RESUMEN}

Objetivos: Describir nuestra experiencia en la implementación de la técnica de intubación submandibular en pacientes con fracturas panfaciales. Materia-

\section{Key words:}

Intratracheal intubation, intraoperative complicactions, airway management, maxillofacial injuries, orthognathic surgery, tracheostomy

\footnotetext{
Médico general, ayudante quirúrgico, Clínica Uros. Neiva, Colombia.

Cirujano maxilofacial, Magister en Tratamiento del dolor. Clínica Uros. Neiva, Colombia.

Especialista en Anestesiología y reanimación. Clínica Uros. Neiva, Colombia.
}

Fecha de ingreso: 03 de diciembre de 2020

Fecha de aceptación: 12 de enero de 2021

\section{ORCID}

0000-0001-6998-3736

Financiamiento: no hubo financiamiento.

Conflicto de interés: todos los autores declaran no tener conflicto de interés

\section{Correspondencia:}

Nelson Daniel Sanchez A. daedra203@hotmail.com 
les y Métodos: Estudio observacional descriptivo, serie de casos de pacientes con reducción quirúrgica de fracturas panfaciales en quienes se aseguró la vía aérea mediante intubación submandibular, desde julio de 2017 a octubre de 2019. Resultados: Se registraron 30 procedimientos de intubación submandibular en 29 hombres y 1 mujer por fracturas panfaciales. Las fracturas del complejo naso orbito etmoidal fueron las más involucradas $(83,3 \%, n=25)$. En la ejecución, el tiempo medio fue de 7,7 minutos (5 a 10 minutos), solo se presentaron 2 casos de ruptura del piloto del balón y desaturación, con rápido reemplazo sin consecuencias. No se registraron complicaciones tempranas ni tardías relacionadas a la herida quirúrgica. Conclusiones: El abordaje submandibular para asegurar la vía aérea es una alternativa con menor dificultad técnica y complicaciones escasas. Para su elección, se debe individualizar el paciente. El equipo quirúrgico deben tener clara comunicación y conocimiento de la técnica.

\section{Palabras clave:}

Intubación endotraqueal, complicaciones intraoperatorias, manejo de la vía aérea, traumatismos maxilofaciales, cirugía ortognática, traqueostomía

\section{Introducción}

E n 1986, Altemir-Hernandez propuso la intubación por vía submandibular (ISM) para procedimientos que requieren fijación intermaxilar, como alternativa a otras técnicas establecidas (traqueostomía, intubacion nasotraqueal)[1]. Indicada principalmente en el manejo quirúrgico de fractuas panfaciales y cirugía ortognática, procedimientos estéticos electivos, de patologias orales y de base de cráneo, siempre que no se requiera o prevea intubación prolongada (mayor a $72 \mathrm{~h})[2]$.

Además, de evitar los potenciales riesgos de la traqueostomía (sangrado complicado, infección, lesión de cuerdas vocales, estenosis traqueal, fístula traqueocutánea y necesidad de cuidados postraqueosotmía) y la intubación nasotraqueal (mayor riesgo de desviación intracraneal en compromiso de la base craneal con afectación de lámina cribosa), son infrecuentes y menores las complicaciones reportadas con esta técnica[2],[3],[4].

Presentamos nuestra experiencia en intubación submandibular en pacientes con fracturas panfaciales programados para reconstrucción quirúrgica.

\section{Materiales y Métodos}

Este es un estudio observacional descriptivo, serie de casos retrospectiva de pacientes admitidos por fracturas panfaciales y programados para reducción quirúrgica, en el servicio de cirugía maxilofacial de la clínica Uros en la ciudad de Neiva, Huila, Colombia, desde julio de 2017 hasta octubre de 2019. Previa aprobación del comité institucional de ética hospitala- ria (Acta No. 54 del 20 de marzo de 2020), se obtuvo reporte de 30 casos en los que se requirió reducción de fracturas panfaciales bajo anestesia general y en quienes se aseguró la vía aérea mediante ISM.

Se realizó la recolección de datos mediante hoja de cálculo de Excel y formato diseñado en Epiinfo 7, donde se hizo el análisis de estadística descriptiva. Como datos de filiación de interés, edad y sexo. Mecanismo de trauma (alta o baja energía) y causas asociadas. Se identificaron las fracturas craneofaciales según el sistema propuesto por Follmar y cols ${ }^{5}$. Se listaron los traumas asociados involucrados según las zonas anatómicas afectadas (craneoencefálico, raquimedular, tórax, abdomen, pelvis y extremidades). Se tuvo en cuenta el tiempo requerido para la ejecución de la ISM, el tiempo quirúrgico para los procedimientos de reducción de fracturas y procedimientos conjuntos en el mismo tiempo quirúrgico según el caso. Asimismo, si presentaba intubación orotraqueal previo al procedimiento, si se logró extubación en el postoperatorio inmediato, extubación programada y si se requirió cuidados intensivos postoperatorios.

\section{Técnica de intubación submandibular}

En la primera fase, bajo protocolos de seguridad de quirófano y anestesia general, se realiza intubacion orotraqueal mediante laringoscopia directa, con tubo endotraqueal reforzado (Tubo Endotraqueal Nasal/ Oral Shiley ${ }^{\top M}$, Medtronic) 7,0 - 8,0 mm de diámetro interno. Previamente se debe tomar la precaución de aflojar el conector que por lo general es resistente a la remoción. Se recomienda posicionar el tubo con relación al primer molar, de preferencia al lado derecho 
para visualizar la acomodación intraoral (Figura 1).

En la segunda fase se realiza intubación submandibular. Previa asepsia y antisepsia de la piel y mucosa oral, se marca el sitio de incisión paralela al eje del cuerpo mandibular (a un través de dedo) entre 1,5 y 2 $\mathrm{cm}$ de longitud, a nivel de la unión del tercio anterior y los dos tercios posteriores del cuerpo mandibular. Con hoja de bisturí $n^{\circ} 15$ se incide piel y tejido celular subcutáneo, con pinza hemostática mediante disección roma se diseca platisma, fascia cervical superficial y musculo milohioideo. Se continua la disección con adherencia al cuerpo mandibular en su superficie interna o lingual, superficial al periostio. Con ello, se evita daño a estructuras como el nervio lingual, las glándulas salivares y sus conductos (Figura 2).

Al lograr relieve en la mucosa oral, con un dedo se desplaza medialmente la emergencia del conducto de Warthon, se realiza incisión intraoral de aproximadamente $1 \mathrm{~cm}$ de longitud con bisturí $n^{\circ} 15$, con lo que se termina de transfixiar y exteriorizar la punta de la pinza. En este momento se libera el tubo del circuito y se retira el conector del tubo. Con la pinza emergida intraoral se toma la punta del balón de control y se exterioriza a través del túnel submandibular. En todo momento se debe sujetar manualmente al tubo para evitar la extubación accidental. En seguida se vuelve a pasar la pinza por el túnel, se toma el extremo del tubo con la pinza emergida intraoral y se exterioriza

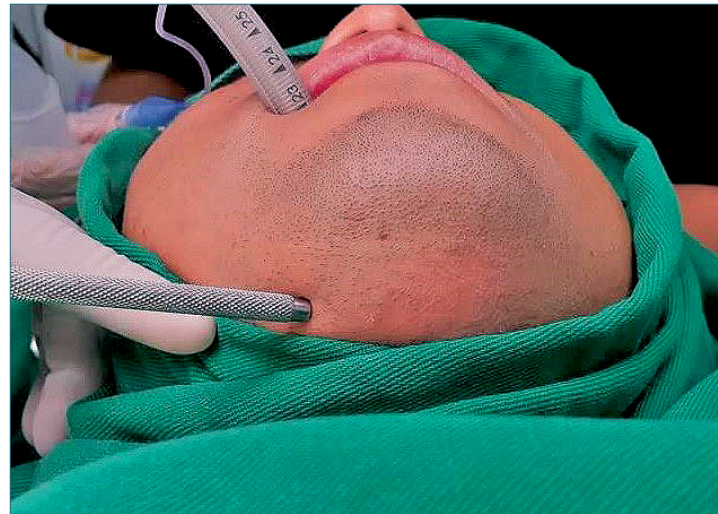

Figura 1. Intubación orotraqueal, selección del sitio de incisión.

a través del túnel. Se revisa que no haya obstrucción intraluminal, se vuelve a colocar el conector, se comprueba la integridad del balón y se conecta de nuevo al circuito. Por último, se comprueba posición intraoral con laringoscopia, capnografía y auscultación bilateral. Se fija el tubo a la piel con sutura de polipropileno 2-0. Al terminar el procedimiento, se devuelve el tubo a la posición oral y se sutura la herida con putos separados de polipropileno 3-0 (Figura 3).

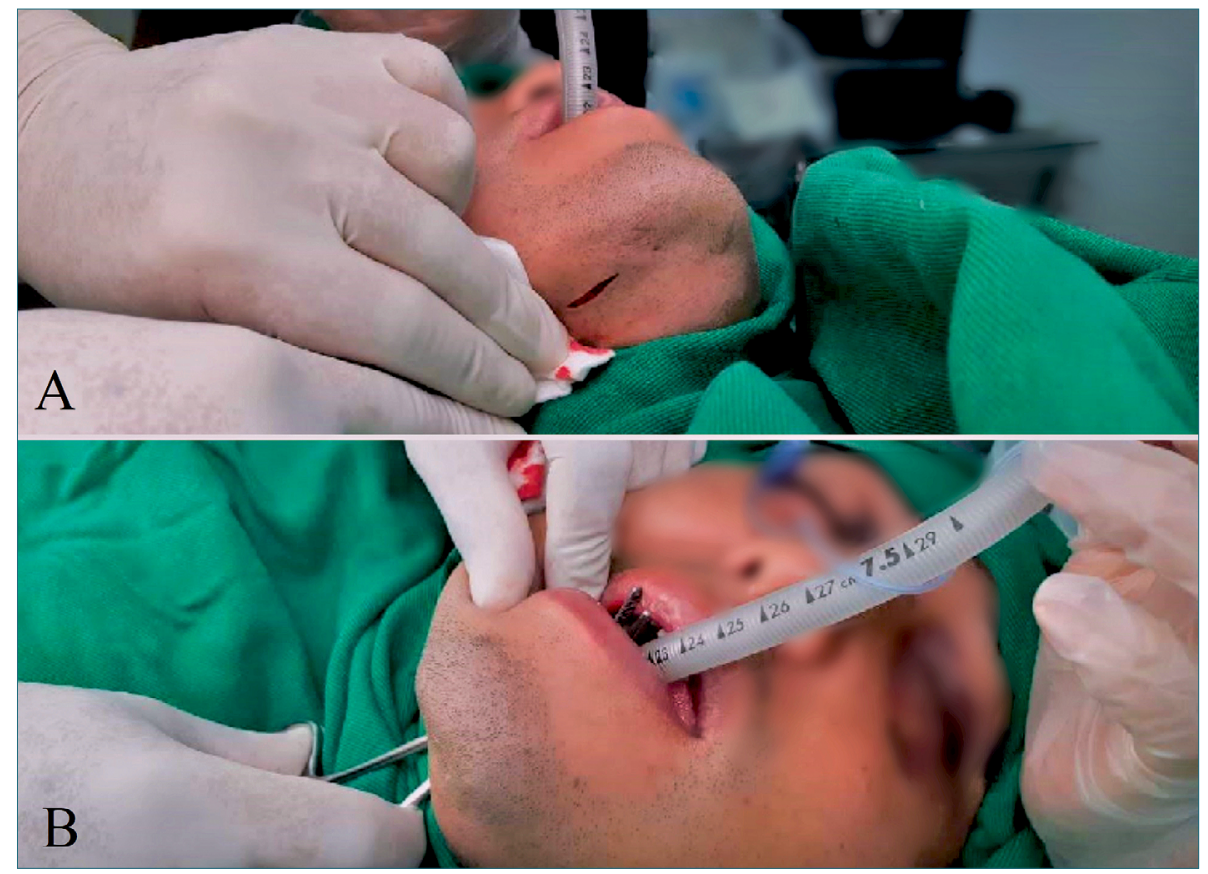

Figura 2. A: Incisión submandibular derecha; B: Disección roma. Previa preparación de la emergencia oral, transfixión a cavidad oral. 


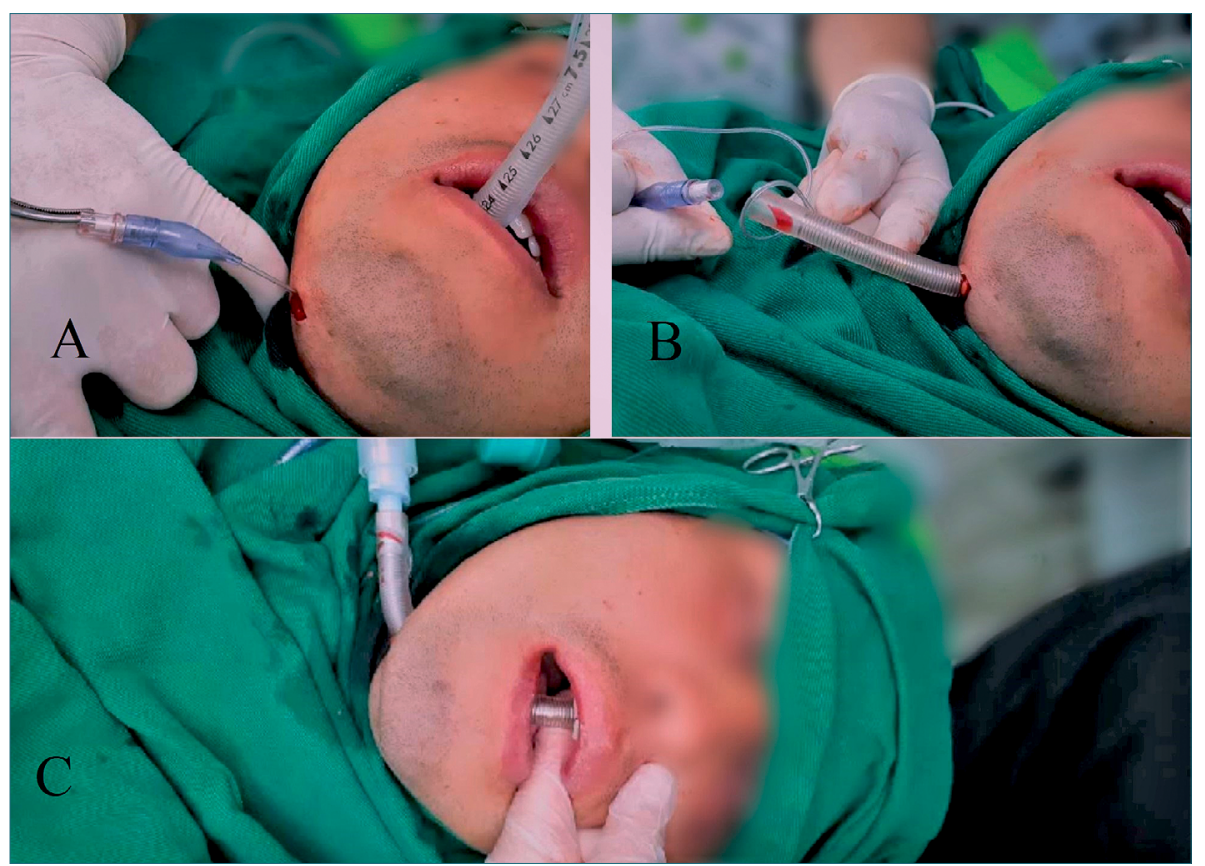

Figura 3. A: Paso del balón de control; $B$. Paso del tubo anillado, sin el conector; C: Conexión al circuito de ventilación. Comprobación de posición del tubo.

\section{Resultados}

Se obtuvo registro de 30 procedimientos de ISM, 29 hombres y 1 mujer, con una media de edad de 26 años (rango entre 17 a 42 años de edad), quienes sufrieron fracturas panfaciales. Veintinueve casos correspondieron a impactos de alta energía relacionados a accidente de tránsito, solo 1 caso de mecanismo de baja energía correspondiente a caída a nivel del suelo.

El tercio facial medio fue el más afectado con presentación en todos los casos, involucrando el complejo naso-orbito-etmoidal en 25 pacientes (83,3\%), seguido de las representadas por fracturas dentoalveolares, frontal, del complejo cigomático malar y mandibulares. Las fracturas faciales involucradas se clasificaron en simples y complejas, según el sistema jerárquico propuesto por Follmar y cols[5] simplificado en la Tabla 1.

Por las condiciones preoperatorias, 8 pacientes $(26,6 \%)$ ingresaron a quirófano con soporte ventilatorio (IOT), 10 pacientes $(33,3 \%)$ requirieron continuar con soporte ventilatorio en su postoperatorio inmediato y solo 1 paciente $(3,3 \%)$ requirió continuar con ventilación mecánica prolongada mayor a 72 h, con posterior realización de traqueostomía. Fue requerido manejo postoperatorio en $\mathrm{UCl}$ en 14 pacientes $(46,6 \%)$. La mayoría de los casos presentaron traumas asociados, de los cuales los traumatismos graves (especialmente craneoencefálico y torácico cerrado) condicionaron las necesidades de ventilacion mecánica pre y postoperatorias. Se resume en la Tabla 2.

La técnica inicialmente empleada para la ISM corresponde a la variación descrita por Stoll[3],[6], con uso de tubo endotraqueal reforzado en todos los casos. El tiempo empleado desde el establecimiento de la intubación orotraqueal, realización de la incisión submandibular, tunelización y paso del tubo hasta el restablecimiento de la conexión a ventilador fue entre 5 a 10 minutos, con media de 7,7 minutos, con tiempo fuera de circuito entre 15 y 35 segundos.

La duración media de los procedimientos quirúrgicos fue de 5,2 h, con un rango entre 2 y 10 h, según la complejidad de las fracturas a reducir y la asociación de lesiones en SNC que requirieron la participación de neurocirugía de manera programada para cierre de fístulas de LCR en 4 casos durante el mismo tiempo quirúrgico.

En cuanto a complicaciones relacionadas al procedimiento de ISM, se presentaron dos casos de ruptura in situ del balón de control antes de iniciar el procedimiento maxilofacial (complicación relacionada al tubo), manifestándose con escape de la ventilación y desaturación leve, rápidamente advertida, requiriendo cambio inmediato del tubo y reposicionamiento, sin presentarse repercusión clínica agregada. No se reportaron complicaciones tempranas ni tardias relacionadas a la herida submandibular. 
Tabla 1. Fracturas involucradas. Adaptado de la clasificación jerárquica según Follmar[6]

\begin{tabular}{llc}
\hline Orden & Fracturas & Resultado \\
Primer orden & Lefort I & $6(20 \%)$ \\
Segundo orden & Lefort III & $9(30 \%)$ \\
Tercer orden & Lefort II & $12(40 \%)$ \\
Cuarto orden & Complejo cigomático malar & $20(66,6 \%)$ \\
Quinto orden (simplificado) & Naso orbito etmoidal & $25(83,3 \%)$ \\
& Fracturas simples: & $20(66,6 \%)$ \\
& - Frontal & $10(33,3 \%)$ \\
& - Orbitaria & $13(43,3 \%)$ \\
& - Nasal & $10(33,3 \%)$ \\
& - Arco cigomático & $10(33,3 \%)$ \\
\hline
\end{tabular}

\begin{tabular}{|c|c|c|c|}
\hline Trauma asociado & $\begin{array}{c}\text { Ventilación mecánica Post } \\
\text { Operatoria }\end{array}$ & $\begin{array}{c}\text { Ventilación mecánica } \\
\text { prolongada (> } 72 \text { h) }\end{array}$ & $\begin{array}{c}\text { Manejo postoperatorio } \\
\text { en Unidad de Cuidado } \\
\text { Intensivo }\end{array}$ \\
\hline Craneoencefálico & $8(26,6 \%)$ & $1(3 \%)^{*}$ & $10^{* *}$ \\
\hline Raquimedular & & & 2 \\
\hline Tórax & & & 2 \\
\hline \multicolumn{4}{|l|}{ Abdomen } \\
\hline \multicolumn{4}{|l|}{ Extremidades } \\
\hline Pelvis & & & \\
\hline
\end{tabular}

*Por pobre modulación de la respuesta inflamatoria; **Tiempo quirúrgico prolongado mayor a 8 horas.

\section{Discusión}

Para la mayoria de los casos de esta serie, el tercio medio fue afectado principalmente por representacion de fracturas incluidas en el primer a cuarto orden (según la clasificación de Follmar[5]) y fracturas simples incluidas en el tercio medio facial que obligaron la fijación intermaxilar, con necesidad de mantener libre el componente nasal, por lo cual, la intubación nasotraqueal no se contempló en muchos casos.

Las fracturas panfaciales comprenden aquellas que involucran al tercio superior, medio e inferior. Por sí mismas, las más involucradas corresponden al maxilar, frontal, complejo naso orbito etmoidal, complejo cigomático y mandíbula, pero los componentes del tercio medio son los más frecuentemente afectados, pues contiene estructuras óseas con menor grado de resistencia a aplicación de fuerzas[7],[8].

Los pacientes con trauma craneoencefálico y cerrado de tórax fueron los que estuvieron relacionados a necesidad de soporte ventilatorio preoperatorio y a continuidad o requerimiento del mismo en el postoperatorio inmediato (craneoencefálico 8 de 26 y torácico 2 de 8 ) con posterior y exitosa extubación programada. Solo un paciente (con trauma craneoencefálico asociado) continuó con ventilación mecánica prolongada y necesidad de traqueostomía, por falla ventilatoria relacionada a una pobre modulación de 
la inflamación sistémica. La duración de los procedimientos por parte de cirugía maxilofacial obedece a la complejidad del trauma. Los de mayor duración fueron los que requirieron intervención conjunta con neurocirugía para manejo de fistulas de LCR en los casos de trauma craneoencefálico severo.

Otros factores que influyen en esta toma decisiones sobre la vía aérea incluyen la presencia de politraumatismo y comorbilidades que hacen prever dependencia a soporte ventilatorio prolongado, por lo cual la necesidad de realizar una traqueostomía para el manejo posterior sería la primera opción. Sin embargo, se deben tener en cuenta las condiciones individuales.

La traqueostomía sigue siendo la técnica estandar en cirugía de fracturas panfaciales, especialmente en el paciente politraumatizado, en quien se prevé soporte ventilatorio prolongado, con requerimiento de vía aérea de emergencia o en el que presenta alteraciones anatómicas importantes que dificulten otras técnicas[9]. Es más complicada en niños, requiere además cuidado postoperatorio especial y aumenta la estancia hospitalaria. Las complicaciones inmediatas, tempranas y tardías son de mayor relevancia clínica, reportadas entre el 5,5\%-7,8\% y mortalidad del $1,4 \%-2 \%[9],[10],[11]$.

El abordaje submandibular permite asegurar la vía aérea a la vez que despeja el campo operatorio y permite una adecuada oclusión dental sin interferencia, tanto el cirujano maxilofacial y el anestesiólogo deben estar familiarizados con la técnica. Originalmente, Altemir propuso el abordaje submentoniano, en posición paramediana, a través del cual se logra una buena acomodación del tubo. Se han publicado múltipes variaciones a la técnica original. Mahmood propuso un abordaje medial retrogeniano, lo que cuida la relación anatómica con el nervio lingual, el conducto submandibular y sus papilas, pero tiene el inconveniente de lesionar la inserción de los músculos geniogloso y geniohioideo, así como la posibilidad de lesión del conducto de Warthon[12],[13]. Stoll y cols., propusieron el abordaje submandibular anterior, logrando una ruta más corta y con menor interferencia en la anatomía muscular excepto por el milohioideo al cual cruza[3],[6].

Otras técnicas descritas tienen sus propias ventajas y desventajas. La vía orotraqueal con técnica retro tuberosa/retromolar requiere paso del tubo anillado posterior al molar maxilar posterior o premolar, fijándolo con sutura circundental, lo que permite la oclusión. Se puede usar aun en fracturas de ángulo mandibular y Le Fort sin interferir en la reducción. Sin embargo, la presencia de una tuberosidad bulbo- sa con poco espacio retromolar o ausencia de molar posterior maxilar con presencia de molar posterior mandibular contraindican su aplicación. Aun así, esta técnica se ha reportado también como rápida y segura. Puede requerir además extracción de tercer molar u osteotomía semilunar retromolar solo para acomodar el tubo, lo que aumentaría el tiempo quirúrgico y afectaría la anatomía ósea[13],[14],[15].

En las revisión sistemáticas de Goh y colaboradores, con 116 estudios desde 1986 a 2018, el tiempo promedio de ejecución de la ISM fue de $10 \mathrm{~min}$ (2-37 min)[2]. Jundt y colaboradores, con 48 estudios desde 1986 a 2011 reportan igual promedio con rango entre 4 y 30 minutos[16]. La serie de Lobo y colaboradores, con 30 pacientes, reporta entre 7 y 10 minutos[17]. Los resultados obtenidos en la presente serie concuerdan con los promedios reportados. Es importante el tiempo en que se ejecuta la ISM por que tiene que ver con la exposicion a desaturacion durante la fase fuera de circuito mientras se pasa el tubo a través del tunel submandibular. También denota la facilidad con la que se puede realizar el procedimiento e indirectamente indica la reproducibilidad en la práctica subsiguiente.

Respecto a las complicaciones en general, la incidencia reportada en las revisiones sistematicas de Goh, Jundt y Lim respectivamente, varian entre 6,8\% a $9 \%$, sin reporte de mortalidad derivadas de la técnica y con todos los procedimientos de ISM llevados a cabo con éxito. Las complicaciones mas frecuentes estan relacionadas a infección superficial $(2,4 \%)$, cicatriz hipertrófica $(0,8 \%)$, formación de fístula mucocutánea o mucocele $(0,11 \%)$ y problemas con el tubo (daño del balón de control) $(0,7 \%)$, migracion selectiva $(0,4 \%)$. Incluso los problemas potenciales como sangrado $(0,17 \%)$, hematoma $(0,13 \%)$, deficit sentitivo motor $(0,08 \%)$, hipoxemia $(0,13 \%)$ u obstrucción $(0,08 \%)$, se reportan en pequeña proporcion pero sin casos de gravedad[2],[17],[18]. En la presente serie, las complicaciones asociadas al tubo endotraqueal (daño del balón de control y desaturación) fueron los únicos hallazgos, sin reporte posterior de complicaciones tardías. Sin embargo, puede existir subreporte de estas últimas dada la naturaleza retrospectiva de la recoleccion de datos.

Las limitaciones del actual estudio se relacionan a su diseño, tratandose de un estudio retrospectivo observacional basado en la recuperación de datos a partir de las historias clínicas de la institución en un periodo de 2 años. Se destaca la homogeneidad de los protocolos implementados en una muestra considerable, siempre por el mismo equipo profesional, lo cual contribuye a la validacion de la técnica. 


\section{Conclusiones}

El abordaje submandibular, cuando está indicado, es una alternativa con menor dificultad técnica, corta curva de aprendizaje, con complicaciones escasas y hasta el momento no asociadas a mortalidad. Inicialmente en pacientes con fracturas panfaciales, especialmente las que involucran del tercio medio e inferior que requieren fijación intermaxilar, también en proce- dimientos electivos como en cirugía ortognática, cirugía de base de cráneo, procedimientos naso palatinos, cirugía de lesiones tumorales de masa importantes en cavidad oral o estructuras de viscerocráneo. La evolución de la técnica ha permitido una disección anatómica con menor riesgo de lesión de estructuras del piso de la boca. Para su elección, el equipo quirúrgico (anestesiólogo y cirujano maxilofacial) deben tener clara comunicación y se debe individualizar el paciente.

\section{Referencias}

1. Hernández Altemir, F. The submental route for endotracheal intubation. A new technique. Journal of Maxillofacial Surgery. 1986 Feb;14(1):64-5. Retrieved from http://www.ncbi.nlm.nih. gov/pubmed/3456416

2. Goh E, Loh N, Loh J. Submental intubation in oral and maxillofacial surgery: a systematic review 1986-2018. British Journal of Oral and Maxillofacial Surgery. 2020;58(1):4350. https://doi.org/10.1016/j. bjoms.2019.10.314

3. Beeram R, Seelam S, Prasad L. Stoll's submandibular intubation: A safety alternative to Altemir's submental technique and traditional tracheostomy. Journal of Oral and Maxillofacial Surgery, Medicine, and Pathology. 2016;28(3):215221. https://doi.org/10.1016/j. ajoms.2015.08.010

4. Kita R, Kikuta T, Takahashi M, Ootani T, Takaoka M, Matsuda $M$ et al. Efficacy and complications of submental tracheal intubation compared with tracheostomy in maxillofacial trauma patients. Journal of Oral Science. 2016;58(1):23-28. https://doi. org/10.2334/josnusd. 58.23

5. Follmar K, Baccarani A, Das R, Erdmann D, Marcus J, Mukundan S. A clinically applicable reporting system for the diagnosis of facial fractures. International Journal of Oral and Maxillofacial Surgery. 2007;36(7):593600. https://doi.org/10.1016/j. ijom.2007.03.010

6. Stoll P, Galli C, Wächter R, Bähr W. Submandibular endotracheal intubation in panfacial fractures. Journal of Clinical Anesthesia. 1994;6(1):83-86. https://doi.org/10.1016/09528180(94)90127-9

7. Greathouse $S$, Adkinson J, Garza R, Gilstrap J, Miller N, Eid S et al. Impact of Injury Mechanisms on Patterns and Management of Facial Fractures. Journal of Craniofacial Surgery. 2015;26(5):15291533. https://doi.org/10.1097/ SCS.0000000000001805

8. Pappachan B, Alexander M. Biomechanics of CranioMaxillofacial Trauma. Journal of Maxillofacial and Oral Surgery. 2011;11(2):224-230. https://doi. org/10.1007/s12663-011-0289-7

9. Cheung N, Napolitano L. Tracheostomy: Epidemiology, Indications, Timing, Technique, and Outcomes. Respiratory Care. 2014;59(6):895-919. https://doi. org/10.4187/respcare.02971

10. Stawicki S, Cipriano A, Mao M, Hon H, Vazquez D, Sharpe R et al. An overview of complications associated with open and percutaneous tracheostomy procedures. International Journal of Critical IIIness and Injury Science. 2015;5(3):179. https://doi. org/10.4103/2229-5151.164994

11. Klemm E, Nowak A. Tracheotomy-Related Deaths: A Systematic Review. Deutsches Aerzteblatt Online. 2017; 10.3238/arztebl.2017.0273

12. Mahmood S, Lello G. Oral endotracheal intubation: Median submental (retrogenial) approach. Journal of Oral and Maxillofacial Surgery. 2002;60(4):473474. https://doi.org/10.1053/ joms.2002.31244

13. Lazaridis N, Zouloumis L, Tilaveridis I, Lazaridou M, Antoniades K, Dimitrakopoulos I. Retrotuberosity versus submentosubmandibular and median submental intubation: patients with maxillofacial surgery. Oral Surgery, Oral Medicine, Oral Pathology and Oral Radiology. 2012;114(5):S209-S215. https://doi.org/10.1016/j. oooo.2011.10.026

14. Jaisani M, Pradhan L, Bhattarai B, Sagtani A. Intubation Techniques: Preferences of Maxillofacial Trauma Surgeons. Journal of Maxillofacial and Oral Surgery. 2014;14(2):501-505. https://doi. org/10.1007/s12663-014-06798

15. Martínez-Lage J, Eslava J, Cebrecos A, Marcos O. Retromolar intubation. Journal of Oral and Maxillofacial Surgery. 1998;56(3):302-305. https:// doi.org/10.1016/502782391(98)90103-3 
16. Jundt J, Cattano D, Hagberg C, Wilson J. Submental intubation: a literature review. International Journal of Oral and Maxillofacial Surgery. 2012;41(1):4654. https://doi.org/10.1016/j. ijom.2011.08.002

17. Lobo Leandro L, García Guevara
$H$, Marinho K, Sanchez Rivero C, Lagunes Lopez M. Intubación submental: experiencia con 30 casos. Revista Española de Cirugía Oral y Maxilofacial. 2015;37(3):132-137. https:// doi.org/10.1016/j.maxilo.2013.12.001
18. Lim D, Ma B, Parumo R, Shanmuhasuntharam $P$. Thirty years of submental intubation: a review. International Journal of Oral and Maxillofacial Surgery. 2018;47(9):1161-1165. https://doi.org/http://dx.doi. org/10.1016/j.ijom.2018.04.015 